\title{
Education and exercise program improves osteoporosis knowledge and changes calcium and vitamin D dietary intake in community dwelling elderly
}

\author{
Ki-Soo Park' ${ }^{1}$ Jun-II Yoo ${ }^{2 *}$, Ha-Young Kim ${ }^{3}$, Sunmee Jang ${ }^{4}$, Yongsoon Park ${ }^{5}$ and Yong-Chan $\mathrm{Ha}^{6^{*}}$
}

\begin{abstract}
Background: Several educational intervention programs have been designed and developed to improve osteoporosis diagnosis and treatment. However, most of the prior studies focused on how educational intervention programs affected diagnosis and treatment of condition of osteoporosis.

The purpose of this prospective and educational intervention study was to evaluate the changes in osteoporosis knowledge, osteoporosis self-efficacy, fall self-efficacy, physical exercise and changes in dietary pattern of calcium and vitamin D intake after osteoporosis education.

Methods: From November 1, 2015 to August 31, 2016, 271 eligible candidates (who were over 50 years old and from 23 different community centers) were recruited through an announcement made by the public office, by two health care providers.

The intervention involved an individualized education program to allow for differences in antecedent educational levels regarding several aspects of osteoporosis, including osteoporosis knowledge, osteoporosis self-efficacy, awareness of self-efficacy risk factors relating to an accidental fall and nutritional education (including the importance of sufficient calcium and vitamin D intake). The researchers revisited the community centers three months after the initial visit.
\end{abstract}

Results: Of the 271 potential participants, 199 (73.4\%; 43 men and 156 women) completed the education program and the second questionnaire. After education intervention, parameters including osteoporosis knowledge, osteoporosis self-efficacy and fall self-efficacy were improved $(P<0.0001)$. After education regarding percentage of calcium and vitamin D intake below recommended cut-offs, inadequate dietary calcium and vitamin D intake were decreased $(P<0.0001)$ from $89.4 \%(178 / 199)$ and $84.4 \%(168 / 199)$ to $79.9 \%(159 / 199)$ and $65.8 \%(131 / 199)$, respectively, at the three-month follow-up. ( $p=0.038, p=0.017$ ).

Conclusions: This prospective intervention study demonstrated that education on osteoporosis knowledge and regular exercise programs could improve osteoporosis self- efficacy, fall self-efficacy and increase dietary calcium and vitamin D intake.

Keywords: Calcium, Education, Exercise, Osteoporosis knowledge, Vitamin D

\footnotetext{
* Correspondence: furim@daum.net; hayongch@naver.com

${ }^{2}$ Department of Orthopaedic Surgery, School of Medicine, Gyeongsang

National University, Jinju, Gyeongsangnam-Do, South Korea

${ }^{6}$ Department of Orthopaedic Surgery, Chung-Ang University College of

Medicine, 102 Heukseok-ro, Dongjak-ku, Seoul 06973, South Korea

Full list of author information is available at the end of the article
}

(c) The Author(s). 2017 Open Access This article is distributed under the terms of the Creative Commons Attribution 4.0 International License (http://creativecommons.org/licenses/by/4.0/), which permits unrestricted use, distribution, and reproduction in any medium, provided you give appropriate credit to the original author(s) and the source, provide a link to the Creative Commons license, and indicate if changes were made. The Creative Commons Public Domain Dedication waiver (http://creativecommons.org/publicdomain/zero/1.0/) applies to the data made available in this article, unless otherwise stated. 


\section{Background}

Osteoporosis, a well-known age-related disease, is a pathology characterized by decreased bone density and regularly results in fractures, which in turn increase the morbidity rate in the elderly population. The pathology is associated with high mortality and socio-economic stress [1-3]. The prevalence of osteoporosis in the general population, aged 50 years or more in Korea, has been reported to be $37.3 \%$ in women and $7.5 \%$ in men [4]. Although osteoporosis is treatable and it is possible to sometimes obviate osteoporotic fractures, approximately $30 \%$ of women will be diagnosed with osteoporosis, and about half of them will require physiciansupervised treatment following a fragility fracture [5-7]. A recent nationwide representative study in Korea has reported that the treatment rate for osteoporosis is only $12.8 \%$, in the general population [4].

In an effort to prevent osteoporotic fractures, several educational intervention programs have been developed to improve osteoporosis diagnosis and treatment. However, most studies were focused on how educational intervention programs affected diagnosis and treatment of osteoporosis in hospitalized patients with hip fractures or other, bone-fragility fractures. Studies such as these (carried out a variety of intervention modalities including telephone call services, education, e-mail, and screening tests for osteoporosis) focused mainly on the improvement of diagnosis and treatment of osteoporosis [8-11]. The hypothesis of this study was the concept that an educational intervention program could improve osteoporosis knowledge, self-efficacy awareness and the dietary habits and customs of the senior population.

Therefore, the purpose of this prospective educational intervention study was to evaluate the relationship between in osteoporosis knowledge, osteoporosis self-efficacy, fall self-efficacy, physical exercise and dietary pattern of calcium and vitamin D intake, following increased awareness arising from increased osteoporosis education.

\section{Methods}

The design and protocol of this study were approved by the Institutional Review Board of our hospital. All patients were informed that his or her medical data might be used in a scientific study. All patients provided consent.

\section{Participants}

From November 1, 2015 to August 31, 2016, 271 eligible candidates (individuals aged 50-95 years without cognitive dysfunction, able to walk, and without malignancy from 23 community centers were recruited through an announcement in the public office by two health care providers. Of these candidates, 40 (14.8\%) were excluded due to an absence of available data necessary to evaluate their dietary calcium and vitamin D intake, 17 (6.3\%) were excluded due to refusal of the first survey, and 15 (5.5\%) were excluded due to refusal of the 2nd survey. After the aforementioned exclusions, a total of 199 participants (43 males and 156 females) were evaluated for the present study (Fig. 1). There were no differences between the characteristics of the 199 participants and those who were excluded.

\section{Educational intervention}

The intervention involved an individualized education program, due to differing levels of education regarding several aspects of osteoporosis including actual comprehension of osteoporosis the disease, osteoporosis self-efficacy, falling self-efficacy risk factors and nutritional education stressing the importance of calcium and vitamin D intake. The participating patients were provided educational materials in print form (brochure developed by the Korean Society for Bone and Mineral Research). The exercise program consisted of stretching for the lower limbs, strength training for the lower limbs, balance training and impact training (all of which could be performed on a self-directed basis at home). All participants were educated in a formal program of therapeutic exercise, initially by a physical trainer and then they were strongly encouraged to keep on with the program, independently, afterwards. The researchers revisited the community centers three months after the initial visit. The consistency of compliance with the exercise program, at the final follow-up, was evaluated using a simple questionnaire. The follow-up reinforced the previously delivered educational messages and determined if any of the study endpoints were reached.

\section{Osteoporosis knowledge questionnaire}

Each participant's knowledge of osteoporosis was assessed using the facts as represented by an osteoporosis quiz (FOOQ) developed by Ailinger et al. [12-15]. These questions were based on the $2001 \mathrm{NIH}$ Consensus Report on osteoporosis. This questionnaire is a validated and psychometrically sound instrument of minimal length that includes questions related to specific risk factors and specific self-care behaviors associated with an osteoporosis diagnosis. The FOOQ is worded at sixthgrade reading level. It has a content validity index of 0.87 and a Cronbach's alpha of 0.76 . The FOOQ consists of 20 true or false questions [16]. In addition to the 'true' and 'false' responses, there was a 'don't know' response option. This third option allowed respondents a choice (for the precise purpose of reducing guessing so that a specific lack of knowledge, "don't know response" and misinformation (incorrect response) could be identified and segregated from each other. In calculating the total raw score for the instrument, each item was assigned a score of " 1 " for a correct answer and a " 0 " if the answer was incorrect or 'don't know'. A total possible score for 


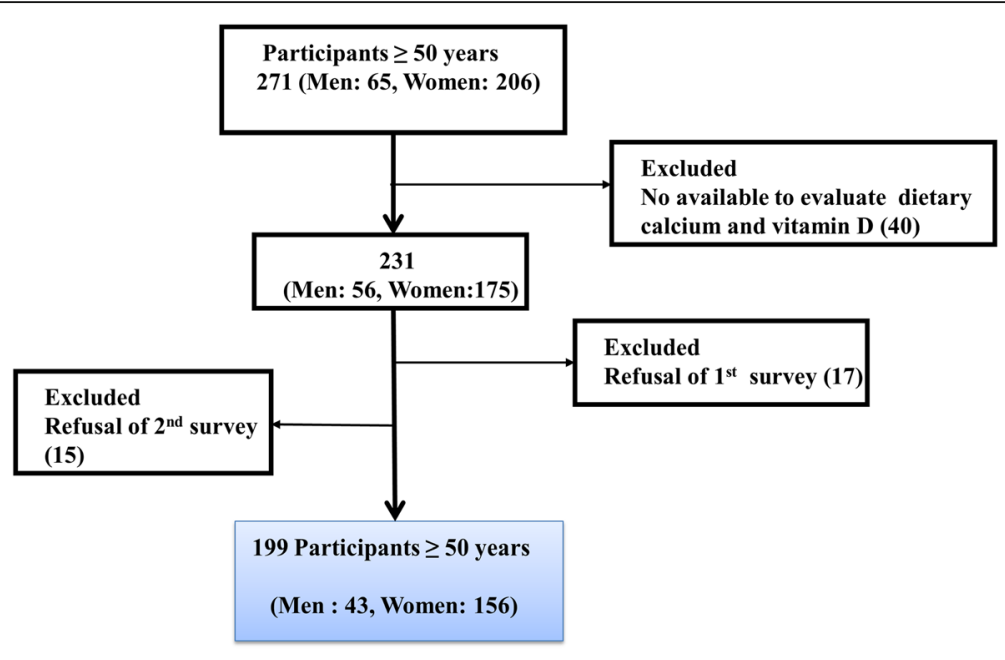

Fig. 1 Flow chart for selection of study participants

the FOOQ ranged from " 0 to 20 ", with higher scores indicating greater comprehension of osteoporosis the disease, and its ramifications.

\section{Osteoporosis self-efficacy questionnaire}

Self-efficacy was measured using a modified 'Osteoporosis Self-Efficacy Scale' developed by Horan et al. [17]. To evaluate osteoporosis self-efficacy, questionnaire derived from research by $\mathrm{Oh}$ et al. [18]. They developed a new questionnaire from that created by Horan et al. [17] by converting it into a form on a scale of 5 points and it underwent verification process. The scale consists of 21 items with two subcategories, exercise (10 items) and calcium (11 items). Each of the 21 items was measured on a five-point scale $(0=$ not at all to $4=$ extremely). Thus, the total score ranged from "0" to " 84 ", with higher scores corresponding to higher osteoporosis self-efficacy.

\section{Fall self-efficacy questionnaire}

The fall self-efficacy questionnaire (Falls Efficacy Scale: FES-I) is a sixteen-item questionnaire developed to assess fall-related self-efficacy based on Falls Efficacy Scale (10 items) [17, 19]. It has six additional items including questions concerning the discharge of more difficult functional tasks and the social ramifications of falling [20]. In order to measure FES-I, questionnaire from research by Jang et al. [21] was used. The sixteen items of FES-I were rated according to "how concerned you are about the possibility of falling" using a visual analogue form anchoring between 0 representing 'not at all confident' (0) and 100 indicating 'Very confident'. Thus, its total score ranged from 0 to 1600 points.

\section{Dietary calcium and vitamin $\mathrm{D}$ intake questionnaire}

The dietary calcium and vitamin D intake questionnaire (Korean Calcium Assessment Tool: KCAT) was developed based on Calcium Calculator ${ }^{\text {th }}$ created by registered dietitians working for the BC Dairy Foundation in the 1980s [22]. The KCAT included seven food groups consisting of 24 categories, 45 food items containing calcium and vitamin D consumed frequently by Koreans. Food items with similar calcium content were put into one category. Average calcium and vitamin D intake was applied according to the Korean Standard Food Composition Tables (8th revision) the daily calcium and vitamin $\mathrm{D}$ intake from fluid according to recommended daily amounts of $700 \mathrm{mg}$. and $15 \mu \mathrm{g}$. in men and $800 \mathrm{mg}$. and $15 \mu \mathrm{g}$. in women, respectively. Frequency of servings was recorded as the number of servings per day, per week, or per month [23].

\section{Statistical analyses}

In the first phase of statistical processing, all variables were assessed using the Kolmogorov-Smirnov test normality of distribution to establish variables' parametric/ nonparametric nature. Because the Kolmogorov-Smirnov test identified all the variables (except AUDIT) as nonparametric (also known as ordinal, descriptive statistics which included calculation of counts and frequencies).

We calculated the required study sample size using the model of Korean Fracture Risk Score [24], in which the mean risk of osteoporotic fracture in IbansumgMeon during sevenyears was $22 \%$. Of risk factors, exercise could reduce $7 \%$ of fracture risk. Based on a power of $80 \%$, significance level of $5 \%$, and the estimated reducing rate of osteoporotic fracture of $7 \%$ in participants, 164 patients were found to be the optimal sample size. Expecting a drop-out rate of 20\% during follow-up, 197 patients were included in the intervention study. Paired 
t-test was used for continual variables. $P$-values were reported two-sided, with $P<0.05$ indicating statistical significance. Statistical analyses were performed using SPSS software version 19.0 (SPSS; Armonk, New York, USA).

\section{Results}

Of the 271 potential participants, 199 (73.4\%; 43 men and 156 women) completed both the education program and the second questionnaire. Of these participants, 159 (79.9\%) were farmers; 162 (81.5\%) graduated from elementary or had no formaleducation. The median age of these participants was 76.3 years (IQR 72-81.5, IQR = Interquartile Range). The median age was 74.9 years (IQR 67.8-81.0) for men and 76.6 for women. (IQR 72.0-82.0) Demographic characteristics of these 199 participants are summarized in Table 1. After education intervention, the osteoporosis knowledge score had increased from a median of 9.1 (IQR 6-13) at preeducation (baseline) to 11.2 (IQR 11-13) at posteducation $(P<0.0001$, Fig. 2$)$. Of these 199 participants, $124(62.3 \%)$ participants had improved osteoporosis knowledge scores while 60 (30.2\%) had worse scores

Table 1 Demographic characteristics

\begin{tabular}{|c|c|}
\hline Number & 199 \\
\hline Men/Women & $43 / 156$ \\
\hline Age (Median) year & $76.3(\mathrm{IQR}, 72.0-81.5)$ \\
\hline Men & $74.9(\mathrm{IQR}, 67.8-81.0)$ \\
\hline Women & $76.6(\mathrm{IQR}, 72.0-82.0)$ \\
\hline BMI $($ Mean $\pm S D)\left(k g / m^{2}\right)$ & $22.4 \pm 3.0($ range, $15.4-32.1)$ \\
\hline Men & $22.5 \pm 3.0$ \\
\hline Women & $22.4 \pm 3.0$ \\
\hline \multicolumn{2}{|l|}{ Education level (school) } \\
\hline Elementary & $162(81.5 \%)$ \\
\hline Middle & $19(9.5 \%)$ \\
\hline High & $15(7.5 \%)$ \\
\hline University & $3(1.5 \%)$ \\
\hline \multicolumn{2}{|l|}{ Occupations } \\
\hline Farmer & $159(79.9 \%)$ \\
\hline Housewife & $15(7.5 \%)$ \\
\hline Employee & $3(1.5 \%)$ \\
\hline Public officer & $1(0.5 \%)$ \\
\hline Business & $4(2.0 \%)$ \\
\hline No works & $16(8.0 \%)$ \\
\hline \multicolumn{2}{|l|}{ Housemates (number) } \\
\hline 1 & $70(35.2 \%)$ \\
\hline 2 & $107(53.8 \%)$ \\
\hline $3-4$ & $20(10.1 \%)$ \\
\hline More than 5 & $2(0.9 \%)$ \\
\hline
\end{tabular}

IQR Interquartile range after the education intervention. The median of the osteoporosis self-efficacy score had improved $(P<$ 0.0001) from 29.6 (IQR 4-52) to 46.1 (IQR 28-64) after the education intervention (Fig. 3).

To determine the exercise efficacy for obviating preventable falls, the median score of fall-related selfefficacy score was noted to have improved $(P<0.0001)$ from 893.0 (IQR 814.3-1360.0) to 1041.8 (IQR 603.81192.5) (Fig. 4).

Although fall-related self-efficacy was improved in 132 $(66.3 \%)$ participants, it was unchanged or actually decreased in $67(33.7 \%)$ of the participants at the time of the three-month follow-up evaluations.

After education regarding percentage of calcium and vitamin D intake below the recommended cut-offs, inadequate dietary calcium and vitamin $\mathrm{D}$ intake were decreased $(P<0.0001)$ from $89.4 \%(178 / 199)$ and $84.4 \%$ $(168 / 199)$ to $79.9 \%(159 / 199)$ and $65.8 \%(131 / 199)$, respectively, at the time of the three-month follow-up. ( $P$ $=0.038, P=0.017$ ).

\section{Discussion}

The objective of this prospective educational intervention study was to determine whether osteoporosis knowledge, osteoporosis self-efficacy, fall self-efficacy, and dietary calcium and vitamin D intake would improve after osteoporosis education and exercise programs.

The principle findings of this study served to demonstrate that when the osteoporosis knowledge score improved from 9.1 to 11.2 , the osteoporosis self-efficacy score improved from 7.4 to 11.5 , and the fall self-efficacy improved from 893 to 1041.8. In addition, patterns, habits and customs of dietary intake in the individual participants were adjusted to improve mean amounts of dietary calcium and vitamin $\mathrm{D}$ intake.

Interventional studies have consistently proven that osteoporosis knowledge, osteoporosis self-efficacy and fall self-efficacy levels all improved after the intervention [25, 26]. Evenson AL et al. [27] have observed the knowledge and awareness of material ramifications of a diagnosis of osteoporosis, health beliefs, self-efficacy, dietary calcium, and vitamin D intakes as measures of preventive behaviors with 153 young adults by using pre- and post- questionnaires including osteoporosis knowledge test, health belief scale, self-efficacy scale, and three-day food records. They reported that knowledge and selfefficacy of osteoporosis were improved after educational intervention. Olsen CF et al. [28] have performed a randomized clinical trial after exercise program and education on the fear of falling among 89 women with osteoporosis and documented history of vertebral fracture diagnosis. They found a significantly better result for the intervention group compared with the conservative group both at three months $(p=0.004)$ and twelve 


\section{Change of Osteoporosis Knowledge}

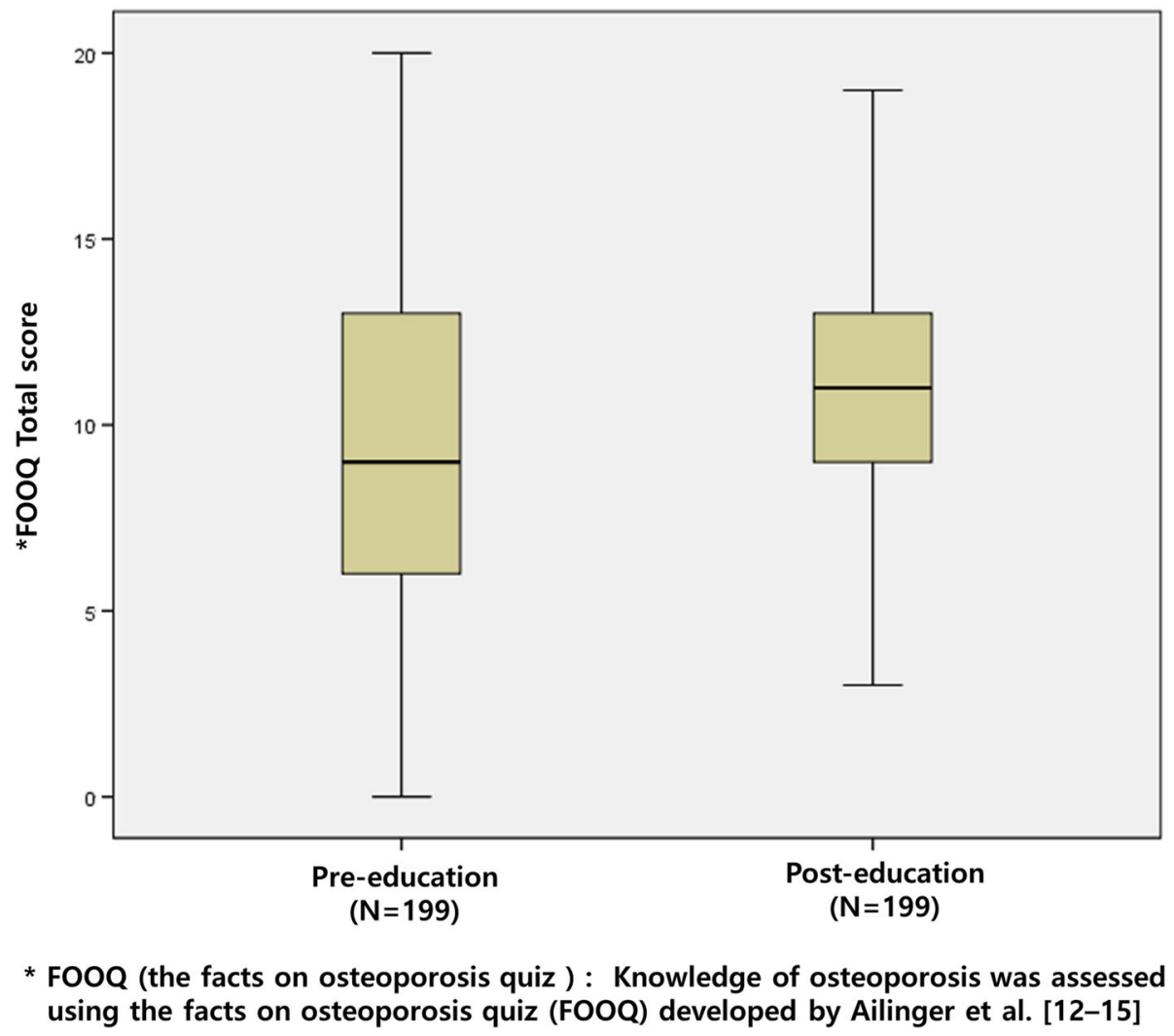

Fig. 2 Change of Osteoporosis knowledge after the education program

months $(p<0.001)$ follow-up. The size of the effect, at three months, was nominal (0.4). However, the effect was moderate (0.7) at twelve months. In this study, we also confirmed effectiveness of education and exercise intervention in community dwelling participants.

Although educational intervention for osteoporosis are frequently focused on BMD and anti-osteoporosis treatments [27, 29, 30], intervention studies regarding changes in life style such as dietary pattern are seldom reported.

Education programs including nutritional education may be effective in increasing dietary calcium and vitamin D intake of community-dwelling populations. Zhao, et al. [31] conducted a prospective randomized control study after nutritional education and dietary intervention for osteoporosis with 90 middle-age and elderly osteoporosis patients. After educational intervention, they found that daily intake of protein, vitamin, calcium, and dietary fiber of the intervention group were significantly higher than those of the control group. Lv and Brown [30] conducted a prospective educational intervention study for increasing intake of calcium rich foods.

After three months of follow-up, they found that the experimental group significantly increased calcium and vitamin $\mathrm{D}$ intake at post-test and such increase was maintained at the time of the follow-up. In this prospective study, we found that education can change food intake patterns, habits and customs, to favor and include food items with high content of calcium and vitamin D. After education intervention, dietary calcium intake increased from $446.8 \mathrm{mg}$. to $537.4 \mathrm{mg}$., and vitamin D intake increased from $8.3 \mu \mathrm{g}$. to $11.7 \mu \mathrm{g}$., consistent with previous findings revealed by previous studies. However, improvement of calcium and vitamin D intake after the intervention study was not sufficient to meet recommendations of calcium and vitamin D intake. These findings are generalized issues in the Korean population. According to the Korean National Health and Nutrition Examination Survey database, mean calcium intake and serum $25(\mathrm{OH}) \mathrm{D}$ level were $485 \mathrm{mg}$./day and $48.1 \mathrm{nmol} . / \mathrm{L}$, respectively in Korean people [32].

Therefore, to improve calcium and vitamin D intake in community dwelling participants, nationwide countermeasures including calcium and vitamin D supplements may be both advisable and necessary.

This study has some limitations. First, the education level and old age of participants made it difficult for 


\section{Change of Osteoporosis Self-efficacy}

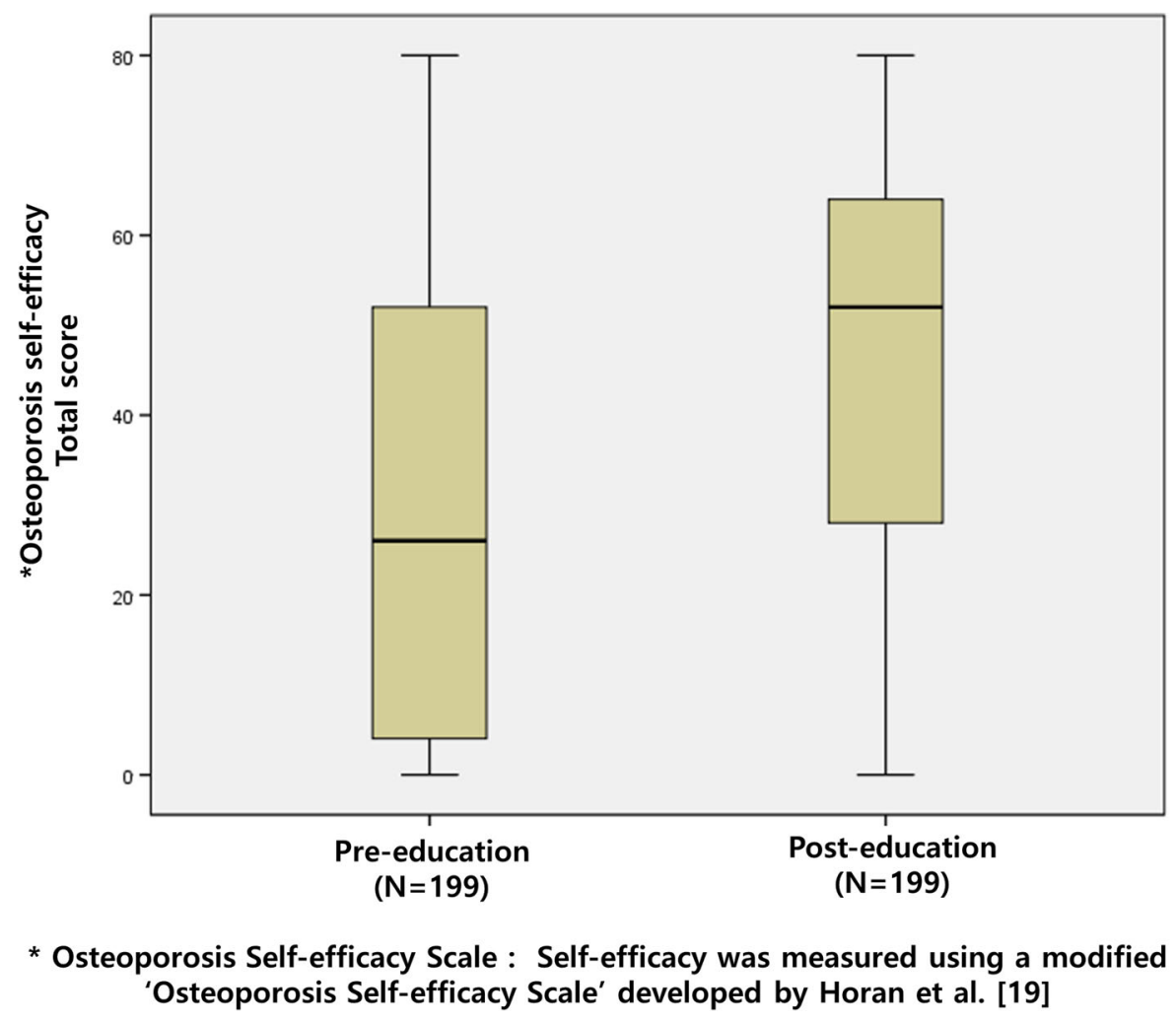

Fig. 3 Change of Osteoporosis Self-Efficacy after the education program

some to understand the effects of osteoporosis knowledge or follow the physical training.

Second, although all participants were provided exercise programs by a physical trainer in one-hour sessions three times weekly, it may not be sufficient to achieve the goal of intervention. Although we could not monitor during the whole period of exercise program, we confirmed the effects of the program without satiable recognition of participants' compliance.

Third, there is no control group in this study and it may be difficult to deduce concrete conclusions about efficacy and impact. Despite these limitations, we hope our results serve as a basis for estimating the positive, long-term effects after intervention, such as increased awareness as to the importance of exercise and education, in other studies.

Fourth, this study could not measure prevalence of osteoporosis and fractures due to limitations of the study design. However, we can confirm improvement of osteoporosis knowledge and self-efficacy and these improvements may have resulted in preventable osteoporosis and related fractures. In addition, the three month study periods in this study could not able to confirm clinically significant effects such as reducing incidence of fall and/ or fracture incidence. Gillespie et al. [33] performed systemic review to assess effect of interventional study such as education including knowledge of osteoporosis, exercise, and nutrient support and improvement awareness, exercise, and using 159 trials with 79,193 participants. They found that exercise programs aimed at reducing falls appear to, in turn, reduce the incidence of osteoporosis-related fractures. Fracture liaison service (which consists of education, screening test for osteoporosis, and pharmacological treatment) is proven to prevent second osteoporotic fractures [34, 35]. In fracture liaison service, patient's education includes improving awareness, exercise, and calcium and vitamin D rich food intake is essential programs [34,36]. Therefore, we think that improved awareness regarding importance of exercise, comprehension of osteoporosis as a pathology, and calcium- and vitamin D-rich food intake in community dwelling participants could induce positive changes in lifestyle, in turn serving to produce decreasing incidents of falls and/or other osteoporotic-related fractures.

However, to confirm a clinically significant effect, longer follow-up study is necessary to further demonstrate a cause-and-effect relationship between improvement of awareness and positive and therapeutic 


\section{Change of Fall Self-efficacy}

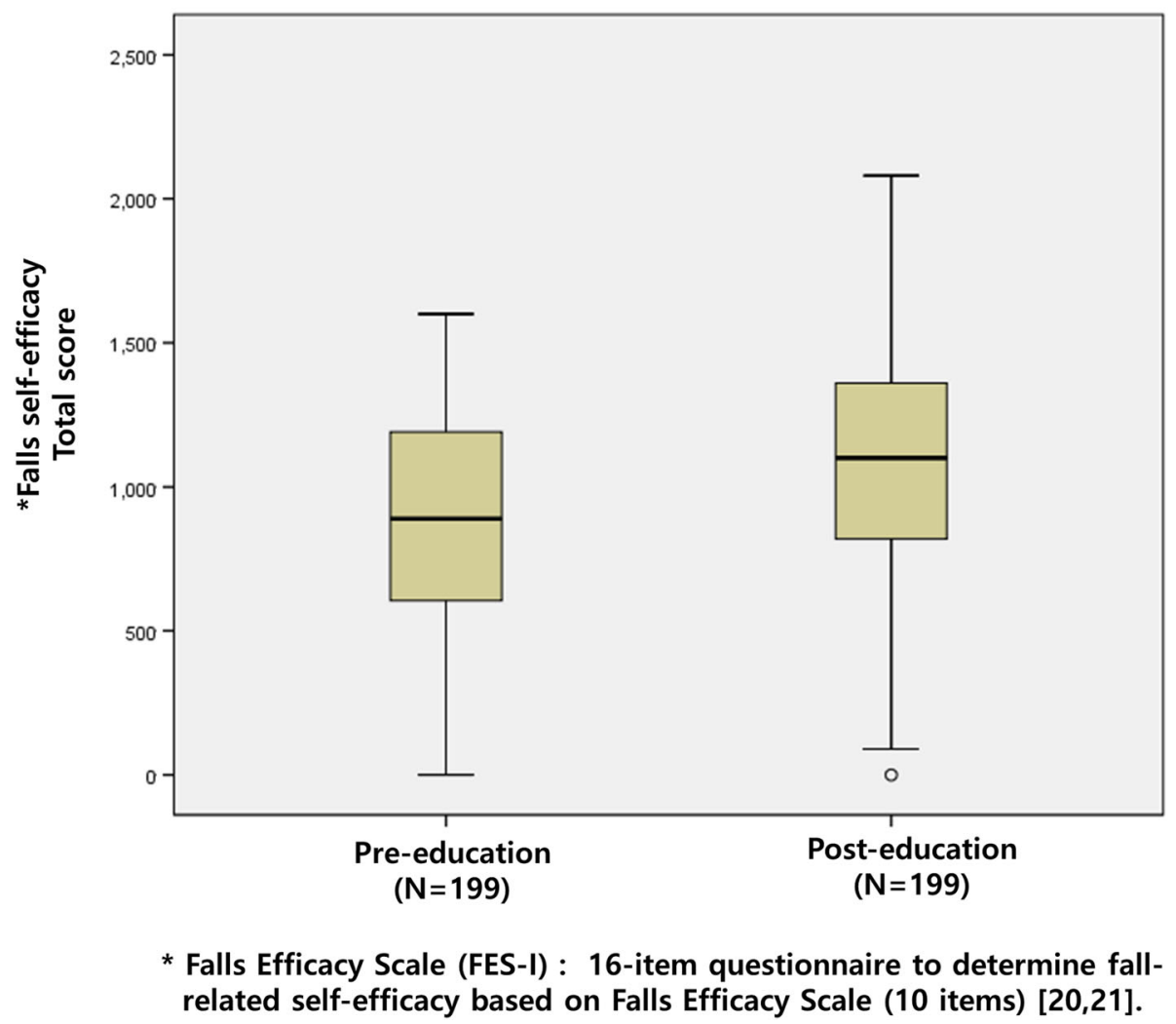

Fig. 4 Change of Fall Self-Efficacy after the education program

changes in life style, and reducing incidents of falling and consequential osteoporotic-related fractures in community dwellings which house an elderly population. Further research will be necessary to identify the relationship between change of self-efficacy and a diminution in fracture rates.

Finally, although calcium and vitamin D intake increased after intervention, the documented increases were insufficient vis-a-vis the recommended intake. In conclusion, follow-up study is felt to be both advisable and necessary to confirm the long-term clinical significance of effects after intervention.

\section{Conclusions}

This prospective intervention study has served to demonstrate that education intervention could improve osteoporosis knowledge, osteoporosis self-efficacy, fall self-efficacy and serve to increase dietary calcium and vitamin $\mathrm{D}$ intake in community dwellings housing elderly populations.

\section{Abbreviations}

FES: Fall efficacy scale; FOOQ: Facts on osteoporosis quiz; KCAT: Korean calcium assessment tool
Acknowledgements

Not applicable.

Funding

Funding for the study was received from the Korea Health Technology R\&D Project through the Korea Health Industry Development Institute (KHIDI) by the Ministry of Health \& Welfare, Republic of Korea. (Grant numbers: HI13C1522, HI15C1189).

This study was supported by a grant of the Center for Farmer's Safety and Health, Ministry of Agriculture, Food and Rural Affairs, Republic of Korea.

\section{Availability of data and materials}

The datasets analyzed in the current study are available from the corresponding author on reasonable request.

\section{Authors' contributions}

KSP and JIY participated in the study design, performed the statistical analysis, and drafted the manuscript; HYK provided assistance in the statistical analysis; KSP, JIY, SMJ performed measurements of variables; YSP and $\mathrm{YCH}$ provided assistance in the literature review and revised the manuscript. All authors read and approved the final manuscript.

\section{Ethics approval and consent to participate}

All subjects provided written informed consent specifically permitting use of their data in the study. The study protocol was approved by the Ethics Committee of Gyeongsang National University Hospital (Approval No. GIRB-A16-Y0012). This study was performed in accordance with the Declaration of Helsinki.

Consent for publication Not applicable. 


\section{Competing interests}

The authors declare that they have no competing interests.

\section{Publisher's Note}

Springer Nature remains neutral with regard to jurisdictional claims in published maps and institutional affiliations.

\section{Author details}

${ }^{1}$ Department of Preventive Medicine and Institute of Health Sciences, Gyeongsang National University Hospital, Jinju, South Korea. ${ }^{2}$ Department of Orthopaedic Surgery, School of Medicine, Gyeongsang National University, Jinju, Gyeongsangnam-Do, South Korea. ${ }^{3}$ Department of Internal Medicine, School of Medicine, Wonkwang University Gunpo Hospital, Gunpo, South Korea. ${ }^{4}$ College of Pharmacy, Gachon University, Incheon, South Korea. ${ }^{5}$ Department of Food and Nutrition, College of Human Ecology, Hanyang University, Seoul, South Korea. ${ }^{6}$ Department of Orthopaedic Surgery, Chung-Ang University College of Medicine, 102 Heukseok-ro, Dongjak-ku, Seoul 06973, South Korea.

\section{Received: 10 February 2017 Accepted: 29 November 2017}

\section{Published online: 19 December 2017}

\section{References}

1. Choi HJ, Shin CS, Ha Y-C, Jang S, Jang S-M, Jang S, et al. Burden of osteoporosis in adults in Korea: a national health insurance database study. J Bone Miner Metab. 2012:30:54-8.

2. Lee S-R, Ha Y-C, Kang H, Park Y-G, Nam KW, Kim S-R. Morbidity and mortality in Jeju residents over 50-years of age with hip fracture with mean 6-year follow-up: a prospective cohort study. J Korean Med Sci. 2013;28:1089-94.

3. Yoon H-K, Park C, Jang S, Jang S, Lee Y-K, Ha Y-C. Incidence and mortality following hip fracture in Korea. J Korean Med Sci. 2011;26:1087-92.

4. Choi YJ, Oh HJ, Kim DJ, Lee Y, Chung Y-S. The prevalence of osteoporosis in Korean adults aged 50 years or older and the higher diagnosis rates in women who were beneficiaries of a national screening program: the Korea National Health and nutrition examination survey 2008-2009. J Bone Miner Res. 2012;27:1879-86.

5. Bessette L, Ste-Marie L-G, Jean S, Davison KS, Beaulieu M, Baranci M, et al. The care gap in diagnosis and treatment of women with a fragility fracture. Osteoporos Int. 2008;19:79-86.

6. Giangregorio L, Papaioannou A, Cranney A, Zytaruk N, Adachi JD. Fragility fractures and the osteoporosis care gap: an international phenomenon. Semin Arthritis Rheum. 2006;35:293-305.

7. Kanis JA, McCloskey EV, Johansson H, Cooper C, Rizzoli R, Reginster J-Y, et al. European guidance for the diagnosis and management of osteoporosis in postmenopausal women. Osteoporos Int. 2013;24:23-57.

8. Heyworth L, Kleinman K, Oddleifson S, Bernstein L, Frampton J, Lehrer M, et al. Comparison of interactive voice response, patient mailing, and mailed registry to encourage screening for osteoporosis: a randomized controlled trial. Osteoporos Int. 2014;25:1519-26.

9. Cook PF, Emiliozzi S, McCabe MM. Telephone counseling to improve osteoporosis treatment adherence: an effectiveness study in community practice settings. Am J Med Qual. 2007;22:445-56.

10. Lafata JE, Kolk D, Peterson EL, McCarthy BD, Weiss TW, Chen Y-T, et al, Improving osteoporosis screening: results from a randomized cluster trial. Gen Intern Med. 2007;22:346-51.

11. Kwon Y-J, Park KS, Choi BH, Kim B-S, Ha Y-C. Prevalence of osteoporosis and effectiveness of screening test using ultrasound bone densitometry and education in a community-dwelling population. J Korean Med Sci. 2016;32:32.e46.

12. Ailinger RL, Harper DC, Lasus HA. Bone up on osteoporosis. Development of the facts on osteoporosis quiz. Orthop Nurs. 1998;17:66-73.

13. Ailinger $R L$, Lasus $H$, Braun MA. Revision of the facts on osteoporosis quiz. Nurs Res. 2003:52:198-201.

14. Park C-H, Lee $\mathrm{Y}-\mathrm{K}, \mathrm{Koo} \mathrm{K}-\mathrm{H}$. Knowledge on osteoporosis among nurses. J Bone Metab. 2017:24:111-5.

15. Yoon B-H, Baek J-H, Lee Y-K, Ha Y-C, Koo K-H. Knowledge on osteoporosis of prescriber according to level of medical institute. Yonsei Med J. 2014:55:1058-62.

16. NIH Consensus Development Panel on Osteoporosis Prevention, Diagnosis, and Therapy. Osteoporosis prevention, diagnosis, and therapy. JAMA. 2001 285:785-95.
17. Horan ML, Kim KK, Gendler P, Froman RD, Patel MD. Development and evaluation of the osteoporosis self-efficacy scale. Res Nurs Health. 1998;21: 395-403.

18. EG O, Yoo JY, Lee JE, Ko IS, Chu SH. Bone health knowledge, self-efficacy, and behaviors in middle-aged Korean women. Korean J Health Promot. 2012;12(2):90-9.

19. Park G, Cho B, Kwon IS, Park BJ, Kim T, Cho KY, et al. Reliability and validity of Korean version of falls efficacy scale-international (KFES-I). J Korean Acad Rehabil Med. 2010:34:554-9.

20. Yardley L, Beyer N, Hauer K, Kempen G, Piot-Ziegler C, Todd C. Development and initial validation of the falls efficacy scale-international (FES-I). Age Ageing. 2005;34:614-9.

21. Jang SN, Cho SI, Ou SW, Lee ES, Baik HW. The validity and reliability of Korean fall efficacy scale(FES) and activities-specific balance confidence scale(ABC). J Korean Geriatr Soc. 2003;7(4):255-68.

22. Hung A, Hamidi M, Riazantseva E, Thompson L, Tile L, Tomlinson G, et al. Validation of a calcium assessment tool in postmenopausal Canadian women. Maturitas. 2011;69:168-72.

23. Food Composition Table, 8th revision [internet]. National Institute of Agricultural Sciences. [cited 2016 Jun 14]. Available from: http://koreanfood. rda.go.kr/kfi/fct/fctPdfDwn/list?totalSearch $Y n=Y$.

24. Kim HY, Jang EJ, Park B, Kim T-Y, Shin S-A, Ha Y-C, et al. Development of a Korean fracture risk score (KFRS) for predicting osteoporotic fracture risk: analysis of data from the Korean National Health Insurance Service. PLoS One. 2016;11:e0158918.

25. EG O, Yoo JY, Lee JE, Hyun SS, Ko IS, Chu SH. Effects of a three-month therapeutic lifestyle modification program to improve bone health in postmenopausal Korean women in a rural community: a randomized controlled trial. Res Nurs Health. 2014;37:292-301.

26. Gaines JM, Marx KA. Older men's knowledge about osteoporosis and educational interventions to increase osteoporosis knowledge in older men: a systematic review. Maturitas. 2011;68:5-12

27. Evenson AL, Sanders GF. Educational Intervention Impact on Osteoporosis Knowledge, Health Beliefs, Self-Efficacy, Dietary Calcium, and Vitamin D Intakes in Young Adults. Orthop Nurs. 2016:35:30-36.

28. Olsen CF, Bergland A. The effect of exercise and education on fear of falling in elderly women with osteoporosis and a history of vertebral fracture: results of a randomized controlled trial. Osteoporos Int. 2014;25:2017-25.

29. Hien VIT, Khan NC, Mai LB, Lam NT, Phuong TM, Nhung BT, et al. Effect of community-based nutrition education intervention on calcium intake and bone mass in postmenopausal Vietnamese women. Public Health Nutr. 2009:12:674-9.

30. LV N, Brown JL. Impact of a nutrition education program to increase intake of calcium-rich foods by Chinese-American women. J Am Diet Assoc. 2011;111:143-9.

31. Zhao C, Zhou R, Tian Y, Tang Y, Ning H, Liu H. Effects of the nutritional education and dietary intervention on nutritional status and bone mineral density of middle-aged and senile patients with osteoporosis. Wei Sheng Yan Jiu. 2016:45:230-5.

32. Joo N-S, Dawson-Hughes B, Kim Y-S, Oh K, Yeum K-J. Impact of calcium and vitamin $D$ insufficiencies on serum parathyroid hormone and bone mineral density: analysis of the fourth and fifth Korea National Health and nutrition examination survey (KNHANES IV-3, 2009 and KNHANES V-1, 2010). J Bone Miner Res. 2013;28:764-70.

33. Gillespie LD, Robertson MC, Gillespie WJ, Sherrington C, Gates S, Clemson $L M$, et al. Interventions for preventing falls in older people living in the community. Cochrane Database Syst Rev. 2012:(9):CD007146.

34. Bogoch ER, Elliot-Gibson V, Beaton D, Sale J, Josse RG. Fracture prevention in the Orthopaedic environment: outcomes of a coordinator-based fracture liaison service. J Bone Joint Surg Am. 2017:99:820-31.

35. Walters $S$, Khan T, Ong T, Sahota O. Fracture liaison services: improving outcomes for patients with osteoporosis. Clin Interv Aging. 2017;12:117-27.

36. Eisman JA, Bogoch ER, Dell R, Harrington JT, McKinney RE, McLellan A, et al. Making the first fracture the last fracture: ASBMR task force report on secondary fracture prevention. J Bone Miner Res. 2012;27:2039-46. 